\title{
Programmable Fluidic Networks Design for Robotic Origami Sequential Self-Folding
}

\author{
Zhenishbek Zhakypov, Mustafa Mete, Julien Fiorentino, and Jamie Paik
}

\begin{abstract}
Self-folding enables compact, deployable and reconfigurable structures for close and far remote applications. As the degree-of-freedom in achieving folded shapes is dictated by the number of actuators, distributed on a quasi-2-D lattice, their design remains challenging in terms of size, weight, efficiency, repeatability and fabrication. While the traditional electric motors are difficult to downscale and assemble, the active materialbased actuators consume high power and are limited by slow and irreversible motions. Here we present a new selective fluiddriven actuation and embedment method for constructing multicreased, self-folding and reversible robotic origami structures. Our design enables an underactuated, yet a distributed control of origami hinges with single and multi-step folding sequence by programming the fluidic networks. The proposed tunable fluidic actuator-channel networks are compactly embedded in a rapid multi-layer lamination process with minimal assembly. We provide an analytic model for the folding actuator unit, validate it with several prototypes and demonstrate its integration into complex networks for folding multi-hinged tessellations, including Miura-ori and sequentially folding box patterns.
\end{abstract}

All authors are with Reconfigurable Robotics Laboratory, Swiss Federal Institute of Technology Lausanne (EPFL), Switzerland.

\section{INTRODUCTION}

$\mathbf{O}$ RIGAMI-inspired self-folding is a key to low-profile and reconfigurable mechanisms and functional structures highly beneficial across a wide range of applications, from micro-drug delivery systems [1] to macro-scale deployable antennas [2] and space solar panels [3]. Recently, self-folding becomes an effective technique for constructing meso-scale robotic origami structures and machines [4], called Robogamis, including reconfigurable sheets $[5,6]$, mechanical meta-materials [7], and self-morphing wings [8]. Researchers developed various actuation methods to enable controlled selffolding by distributing electromechanical motors [9], thermally activated materials, such as shape memory alloys (SMA) [10], shape memory polymers (SMP) [11] and hydrogel [12], heated locally employing resistive elements [13], or externally by means of ovens [14], light [15, 16], and even laser irradiation [17]. Among these solutions, electrical motors provide a high positioning accuracy, however, they generate limited torques at meso-scale that hinders their application for
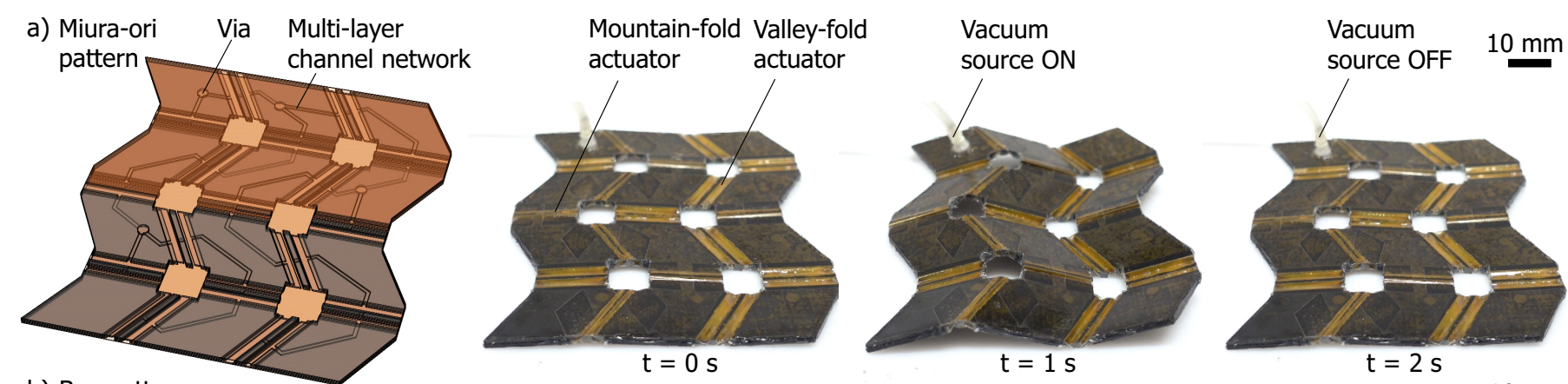

b) Box pattern
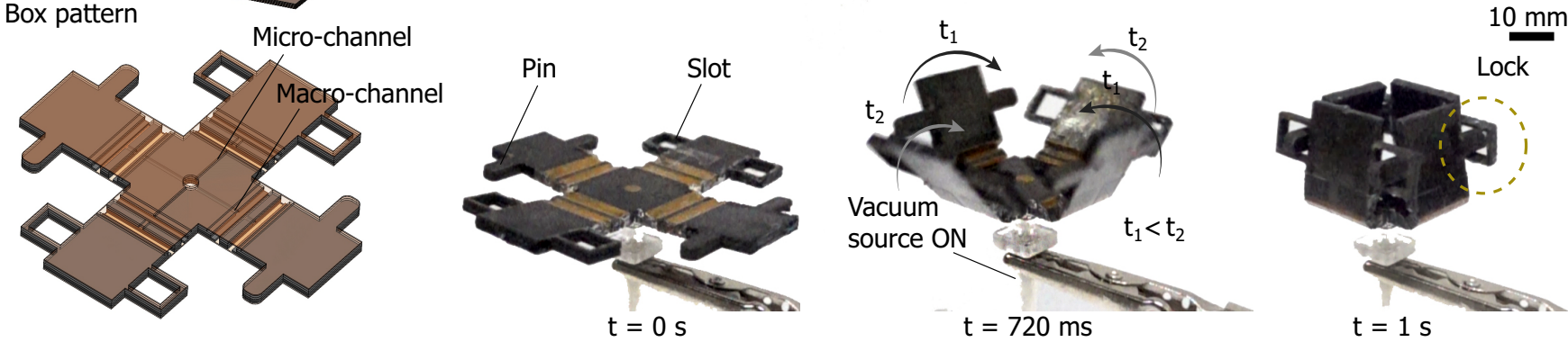

Fig. 1. Two examples of reconfigurable self-folding origami structures driven by the proposed fluidic actuator-channel networks. a) The Miura-ori pattern embeds two rigid channel layers to distribute pressure among the mountain and valley folding actuators. The direction of folding is decided by the gap size on the top and bottom rigid cover layers. b) The box pattern with four walls folding in a programmed sequence. Two walls embed a micro-channel with cross-sectional dimensions of $0.125 \mathrm{~mm} \times 0.5 \mathrm{~mm}$ and the other pair has a macro-channel with dimensions $0.5 \mathrm{~mm} \times 1 \mathrm{~mm}$. The difference in the channel size results in a folding time delay occurring at high speeds $(\leq 200 \mathrm{~ms})$. We introduced pin and slot connectors on the walls to ensure locking only in the right folding sequence; first pin then slot, and to display the delay visually. 
multi-creased origami patterns. Thermally-activated functional material-based actuators, on the other hand, overcome some of these challenges being highly customizable and compact for embedment by multi-layer lamination processes, however, they are still substantially slow $(<1 \mathrm{~Hz})$, inefficient and irreversible without using an antagonistic pair, which further inhibits actuation. Some research have shown high speed and repeated folding of origami hinges by employing discrete inflatable pouch [18] and elastomeric [19] actuators. However, the high volumetric expansion of the actuators $(>200 \%)$ limits their distribution density and motion range for more complex multihinged patterns, like the Miura-ori tesselation in Fig. 1, which has several mountain and valley hinges connected at a single vertex. Moreover, such air-pockets require a pre-fabrication process, such as heat sealing or molding, before integrating into a robotic origami. They were implemented in limited numbers for moving pre-folded shape changing origami structures [20] with individual fluidic piping. Similarly, a vacuum-driven actuator has been proposed [21] for collapsing readily-folded origami structures by encapsulating them in an airtight film, which lacks 2-D to 3-D self-folding capability and control over the folding sequence.

In this paper, we present a method for designing single source, progammable, fluidic actuator and channel-embedded networks for origami sequential self-folding. The proposed low-profile and at scale vacuum-powered multi-layer networks enable designs of complex multi-hinged origami patterns with fast $(>1 \mathrm{~Hz})$ folding and reversible shape reconfiguration. Owing to tunable design combined with layer-by-layer rapid manufacturing process, our approach allows for selective embedment of actuators and channels for folding complex origami patterns with mountain and valley folds in one step, like the Miura-ori tesselation in Fig. 1a, or in a sequence, like the box pattern with tunable channels in Fig. 1b. The main contributions of our work are

- The novel distributed fluidic actuator design, modeling and embedment method for repeated robotic origami selffolding.

- The programmable design of underactuated fluidic networks for controlling folding sequence.

- Experimental validation of the proposed folding actuator model and networks by several prototypes.

\section{Fluidic Origami Networks Design}

In this section, we present the design principle and analytic model of the proposed fluidic actuator-channel networks to achieve origami sequential self-folding.

\section{A. Actuator Requirements for Self-folding Origami Structures}

Actuators play critical roles in active self-folding structures. Besides being compact and lightweight to allow for distribution of several on a quasi-2-D lattice without overloads, the actuators should also be customizable and reversible in terms of force and motion to achieve flexibility in shape transformation and movement. The actuator solutions presented in the literature are often embedded as independent layers or

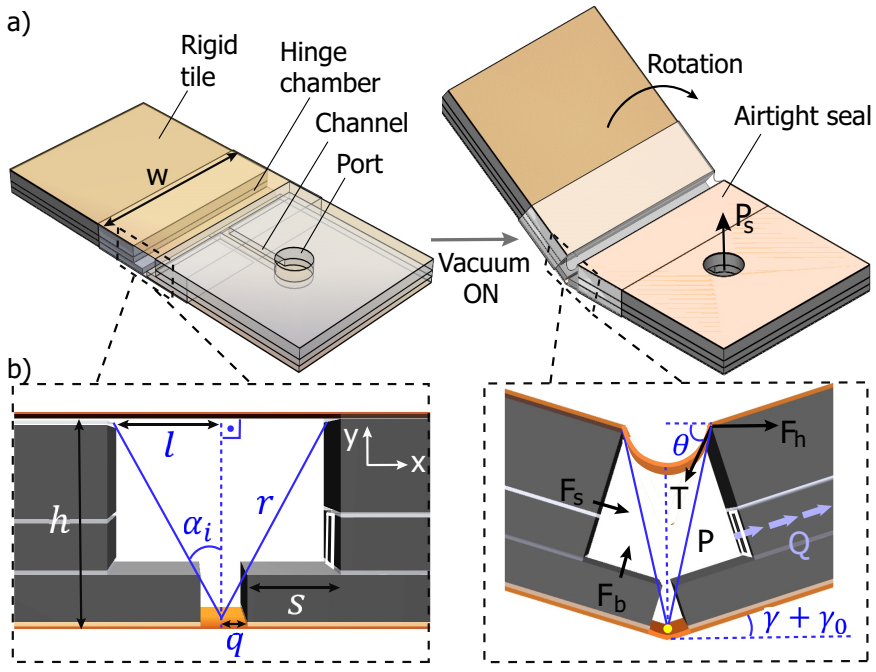

Fig. 2. The actuator-channel unit design that generates bending motion upon applied vacuum pressure. a) The actuator hinge embeds an empty chamber between two rectangular rigid parts, sealed by flexible membranes on four sides. Bending is produced due to the surface area difference between the top and bottom membranes; the top membrane possesses larger area compared to the one at the bottom, therefore, it collapses at vacuum pressure, generating a tension force. b) The forces produced by the negative pressure acting on the membrane and rigid walls generate a bending moment at the yellow pivot.

discrete components onto the flexible hinge and rigid tile layers. Therefore, the actuator power-to-weight ratio becomes a crucial factor in defining the performance as they carry the neighboring actuator-hinge-tile branches. The vast majority of existing studies considered one-way hinge folding that inhibits repeatability and hence re-usage. We propose a vacuumpowered fluidic actuator unit depicted in Fig. 2a that addresses these challenges. Made of an air chamber encapsulated by a thin compliant membrane, our actuator is nearly weightless and compact, embedded between rigid tiles. The operation principle and model of the actuator are presented next.

\section{B. Fluidic Actuator Unit Design}

1) Force and Motion Generation: The fluidic actuator and channel-embedded unit consists of a T-shaped void or chamber between two rigid tiles and airtight deformable membranes covering its four sides: top, bottom and two side walls, as in Fig. 2a. A channel with rectangular cross-section residing close to the bottom enables air flow between the pneumatic source and the actuator chamber. The hinge bending occurs upon applied vacuum pressure, due to the surface area difference between the top and bottom membranes, with latter possessing a smaller surface area. The force produced by the difference between the high atmospheric pressure outside and low pressure inside the chamber deforms the top membrane inward and pulls the side and bottom rigid walls, generating a bending moment at the middle of bottom membrane (yellow dot).

To establish a clear relation between the actuator geometry, membrane stiffness and fluid dynamics and it output moment and bending angle, we studied the physical model, given in 
Figure $2 b$. The deformation of the membrane is approximated by a planar cable deforming under distributed load [22], whereas the bottom membrane experiences pure bending. We neglected the effect of the side membranes due to complexity of predicting their deformation. The deflection of the top membrane under applied pressure can be expressed by

$$
y=\frac{1}{F_{h}} \iint \Delta P w d x^{2}=\frac{\Delta P w x^{2}}{2 F_{h}}
$$

here, the distributed load $\Delta P=P-P_{a t m}$ acts on the top membrane surface spanning $x-z$ Cartesian plane with width $w$ and generates horizontal force $F_{h}$. Now, by taking the derivative of the expression (1) in $x$, we can compute the slope of the curve as

$$
\frac{d y}{d x}=\tan \theta=\frac{\Delta P w x}{F_{h}}
$$

We also know that the length of the curved membrane is $l=\frac{1}{2} \int_{-l}^{+l} \sqrt{1+\left(\frac{d y}{d x}\right)^{2}} d x$, so we can find the horizontal force $F_{h}$, which relates to the tension force by $T=\frac{F_{h}}{\cos \theta}$. The applied pressure similarly produces forces at the side and bottom rigid walls $F_{s}$ and $F_{b}$, respectively, that are the product of the pressure and their surface areas

$$
F_{s}+F_{b}=\Delta P w(h+s)
$$

where $h$ is the thickness of the actuator and $s$ is the width of the protruding rigid layer at the bottom. We can now calculate the moment produced by the applied pressure at the bottom pivot (yellow dot) as follows

$$
M_{p}=r T \cos \left(\alpha_{i}-\gamma-\gamma_{0}+\theta\right)+\frac{h F_{s}}{k}+\frac{F_{b}}{\frac{s}{2}+q}
$$

here, $\alpha_{i}=\arctan \frac{l}{h}$ is the actuator initial angle before actuation, $\gamma$ and $\gamma_{0}$ are the half bending and recovery angles of the bottom membrane, $\theta$ is the top membrane bending angle, $q$ and $l$ are the half lengths of the bottom and top hinges, respectively. $F_{s}$ generated by the side wall is separated into two components that contribute to the bending of both membranes and their proportion is a function of the membrane stiffness $1 / k$. Although, we neglect the membrane weight and assume the moment is generated solely by pressure, the membrane stiffness that counters the moment is crucial in defining the actuator output moment, which is described next.

2) Hinge Membrane Stiffness Model: The stiffness of the membrane is critical for force generation and hinge restoration. We assume that the proposed actuator hinge comprising two membranes deforms elastically and undergoes pure bending. For a bending sheet, the moment is given by $M=E I \phi=$ $E \frac{\text { Width } \times(\text { Thickness })^{3}}{12 \times \text { Length }} \phi$, where $E$ is the elastic modulus of the material, $I$ is the second moment of inertia and $\phi$ is the bending angle.

In order to sum the effect of both membranes, the bending moments of the bottom and top membranes are calculated about the bottom membrane's center. The total bending moment resisting to actuation can be calculated by

$$
M_{k}=E \frac{w(t)^{3}}{12}\left[\frac{\gamma+\gamma_{0}}{q}+\frac{\theta}{l^{2}} r \sin \left(\alpha_{i}-\gamma-\gamma_{0}+\theta\right)\right]
$$

The actuator total moment can now be calculated as $M_{t}=$ $M_{p}-M_{k}$. Increasing the cross-sectional area of the actuator increases force and placing multiple chambers in series increases its angular range, as in Fig. 1b.

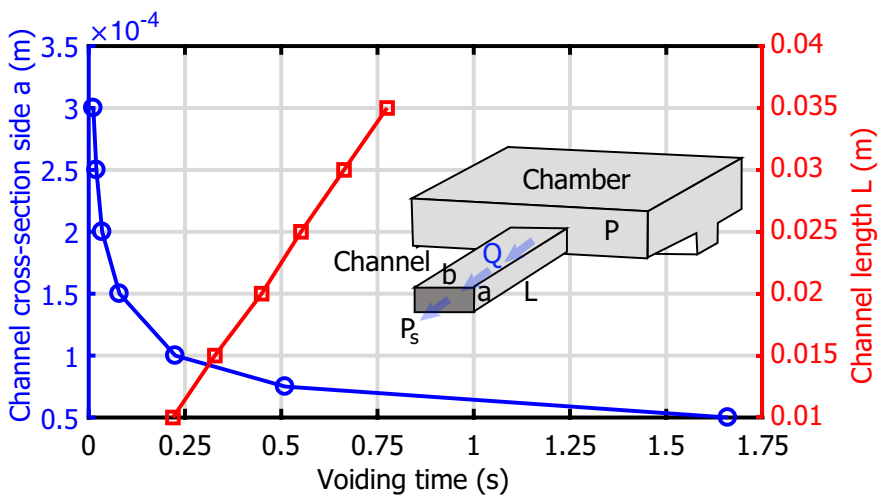

Fig. 3. The relation between the air voiding time and channel geometry for a chamber with fixed volume. Air flow rate drops (blue) significantly for a smaller channel size, where it reduces linearly (red) with channel elongation. This highlights the ability of varying the fluidic resistance to design a variable time delay networks.

3) Quasi-Static Fluid Model for Folding Sequence Programming: To achieve flexibility in realizing various selffolding origami structures, it requires not only a proper arrangement of crease patterns but also control over their folding sequence. We propose a single pressure source underactuation method for folding sequence control of distributed actuators by tuning the resistance of fluidic channels, therefore, programming the folding sequence logic circuitry into the active multi-creased origami structures.

For a rectangular fluidic channel carrying a laminar flow, the volumetric flow rate is given by

$$
Q(t)=\left(P(t)-P_{s}\right) \frac{\rho(t)}{\mu} \underbrace{\frac{a^{3} b}{12 L}\left[1-0.630 \frac{a}{b}\right]}_{R_{c h}^{-1}}
$$

where $Q$ is the volumetric flow rate, $P(t)$ and $P_{s}$ are the hinge chamber and source pressures, $\rho$ and $\mu$ are the air density and viscosity, respectively, and $R_{c h}$ is the channel resistance, which relies on the channel cross-sectional dimensions $a$ and $b$, and its length $L$. Clearly, a channel with larger crosssectional area permits higher flow rates and the variation is more significant for channel cross-sectional dimensions than for channel length. Now, if we assume a locally steady flow that obeys the ideal gas law, the air density inside the chamber evolves as follows

$$
\rho(t+\Delta t)=\rho(t)\left[1-\frac{Q(t)}{V_{T}} \Delta t\right]
$$




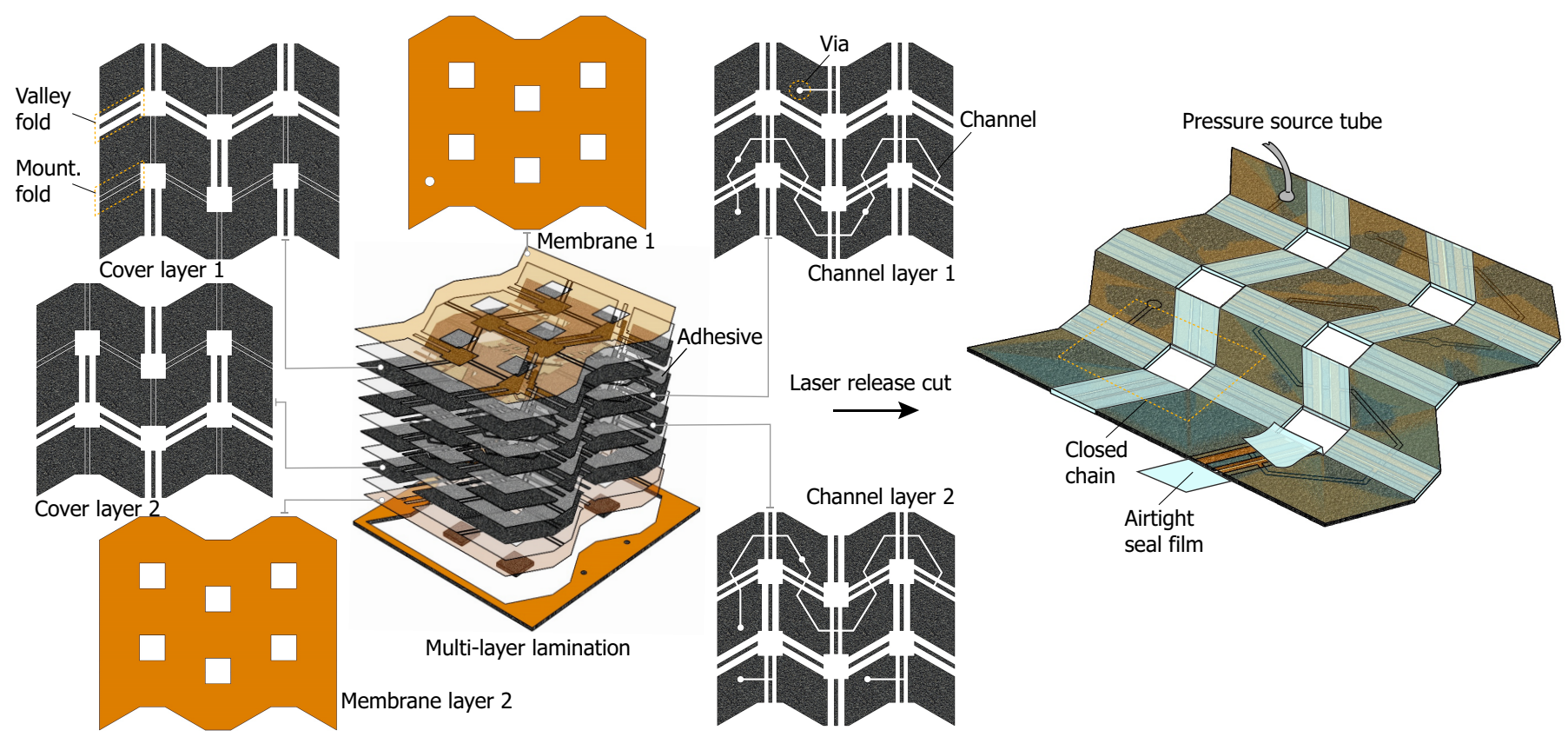

Fig. 4. The layer-by-layer fabrication and assembly process of the fluidic actuator-channel networks for origami self-folding. Here, we illustrate the fabrication process of a well-known Miura-ori crease pattern as an example. The 2-D structure consists of 11 layers of rigid, flexible and adhesive layers. As the structure requires folding in mountain and valley, we designed two channel layers interconnected by vias to maintain air flow between the layers. First, the discrete layers are laser cut separately in rectangular forms with grooves for actuator chambers and channels. Then the layers are stacked and aligned by cylindrical pins on a metallic plate and heat pressed. The sandwich is then laser cut again to a desired outline to release hinges from the supports. Finally, we air-seal the hinges with laser cut hot melt films by wrapping around the chambers and heat pressing.

where $V_{T}$ is the total air volume inside the hinge chamber and channel combined before actuation, and $\Delta t$ is the time step. Then the pressure changes with density as $P(t)=R T \rho(t)$, where $R$ is the air specific gas constant and $T$ is the temperature. The complete voiding of the hinge chamber occurs with $Q\left(t_{f}\right)=0$ at time $t_{f}$.

We simulated the relations in (6) and (7) in Matlab and compared the voiding time for different channel cross-sectional dimensions and length in Fig. 3. We assumed a fixed volume chamber and run the simulation until the flow of air particles diminishes. The flow drops significantly with a slight decrease of the cross-sectional side $a$, whereas it is linearly proportional to the channel length.

\section{Multi-Layer Fluidic Networks Design}

The fluidic folding actuator unit enables integration into large networks to achieve complex origami shapes by multilayer design process. To demonstrate the versatility of our design method, we pick a well-known, yet challenging pattern Miura-ori, illustrated in Fig. 4, which possesses both mountain and valley folds connected in closed chains. We designed a pattern with 17 fluidic folding actuator units ( 9 valley and 8 mountain) connected in a large network via channels. This requires six functional layers to achieve self-folding: a pair of rigid cover, two rigid channel and two flexible membrane layers. The gap size on the cover layers decides mountain or valley folds: the actuator produces a valley fold if the top cover gap is larger than the bottom one and a mountain fold if vice versa. To employ a single source and distribute the pressure among the actuators it requires two channel layers; one for the mountain folds and another for the valley folds. Like with electrical circuits, the connection between the channel layers can be maintained by vias. The top and bottom flexible membranes make the hinges, ensuring sealing, structural integrity of the rigid parts and shape recovery. We employ channels with large cross-sectional areas (macro-channels), however, one can also design multi-layer channel networks with variable channel size to achieve sequential folding, like in the folding box example in Fig. 1b.

\section{Multi-Layer Fluidic Networks Fabrication}

In this section, we provide the rapid multi-layer fabrication and assembly process of the proposed actuator-channel fluidic networks.

\section{A. Fluidic Networks Fabrication}

The proposed fluidic networks comprise channels with various cross-sectional dimensions varying from few hundred micrometers to few millimeters. Employing 3-D printers is not a viable option as their resolution is limited and it is difficult to achieve complex multi-layer fluidic networks without employing a support material. Therefore, we employed a layerby-layer lamination process depicted in Fig. 4 to readily embed actuators and channels into multi-tile origami structures with high flexibility.

Fig. 4 shows the fabrication steps of the Miuri-ori pattern. The quasi-2-D structure comprises six functional and five adhesive layers. We employ $500 \mu \mathrm{m}$ thick FR4 fiberglass 


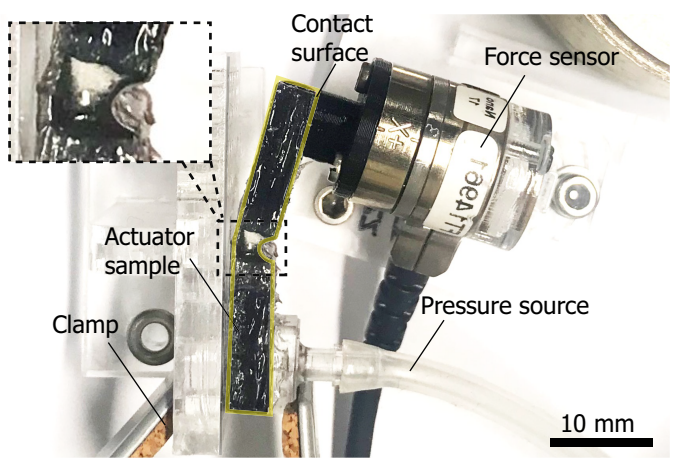

(a) Force measurement setup

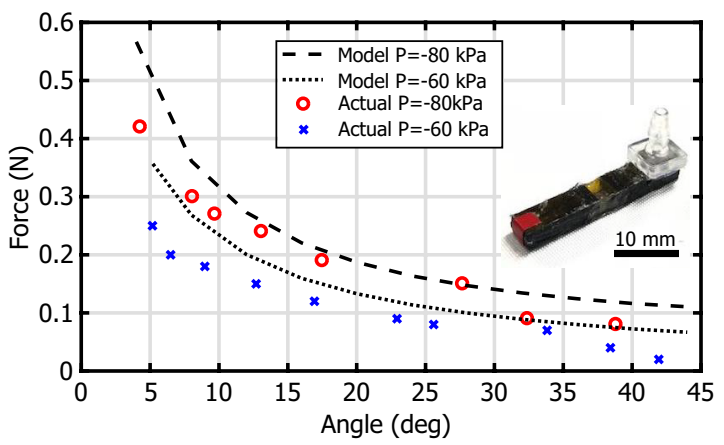

(c) Force vs angle for sample 2 thick-narrow

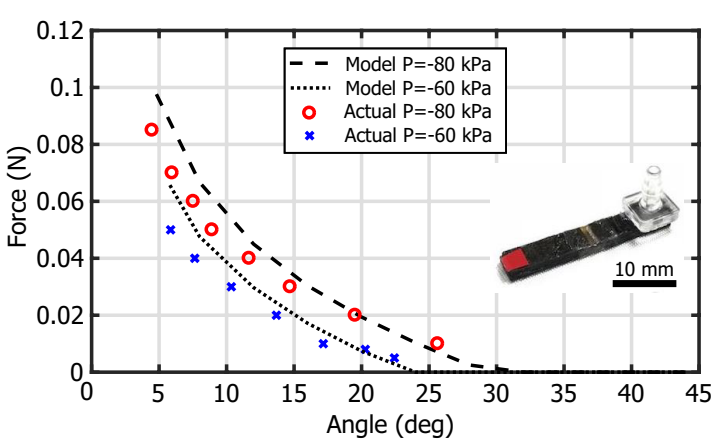

(b) Force vs angle for sample 1 thin-wide

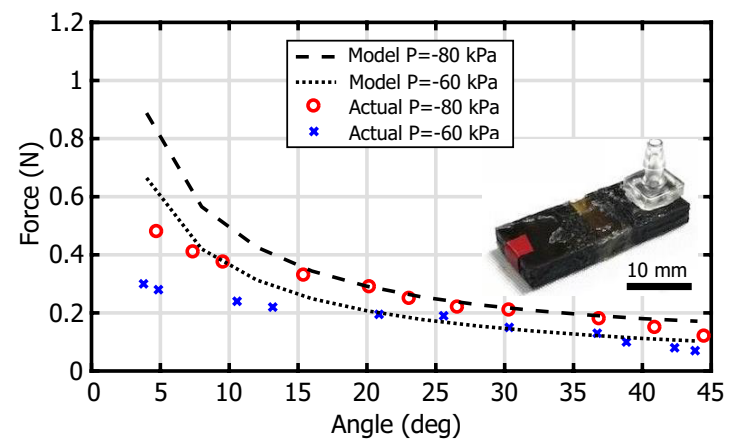

(d) Force vs angle for sample 3 thick-wide

Fig. 5. The experimental setup and results for the blocked force tests of actuator samples with different geometrical parameters at different angles. a) The experimental setup comprises a force sensor, a camera for measuring angles and the actuator sample with fixed one end. The force is measured by stationing the sensor in contact with the actuator moving arm at different angles and pressurizing the actuator. We assessed the forces generated by the actuator samples with the following chamber sizes: b) $2 \times 2.2 \times 7 \mathrm{~mm}^{3}$, c) $4 \times 4.5 \times 7 \mathrm{~mm}^{3}$ and d) $4 \times 4.5 \times 15 \mathrm{~mm}^{3}$.

sheet for rigid tiles due to lightweight and high stiffness characteristics, whereas the membranes are made of a $25 \mu \mathrm{m}$ thick polyimide film (Kapton, DuPont Inc.) due to durability. Poli-melt 701 hotmelt adhesive (Poli-Tape Group) is employed for bonding the layers together, as well as for air-sealing the actuator chambers by wrapping around. The fabrication process is as follows: first, the individual layers are cut employing a laser micro-machining station (Speedy400, Trotec Laser $\mathrm{GmbH}$ ) in rectangular forms with necessary grooves for actuation and channels then they are stacked on a metal plate by aligning with pins. The multi-layer stack is then heat pressed at $110{ }^{\circ} \mathrm{C}$ for eight minutes to ensure layer bonding. The sandwich then is laser cut again to release hinges from the surrounding support material. As the final structure lacks side membranes, we wrapped the laser cut hotmelt adhesive strips around the hinges and heat pressed again at $110{ }^{\circ} \mathrm{C}$ for three minutes. This enables the hinge side walls sealing, however, we applied an additional thin layer of silicone glue (Sil-Poxy $\mathrm{TM}$, Smooth On Inc.) on the seal conjunctions and fiberglass edges to ensure full air impermeability.

\section{EXPERIMENTAL RESULTS}

To assess and validate the models presented in Section II across several samples with different geometry, we measured blocked forces for each when actuated at different angles using the setup depicted in Fig. 5a. The setup comprises a force sensor (Nano 17, ATI Instruments), the actuator sample fixed at one end and in contact with the sensor at the other end, a camera mounted above the sample to extract the actuator angles using image processing software (Image $\mathrm{J}$ ) and a vacuum pressure source with a pressure indicator sensor (SMC Corp.) connected to the actuator via a $3-\mathrm{mm}$ diameter pipe. In our experiments, vacuum is achieved by converting a positive pressure using a vacuum ejector (ZH10BS-06-06, SMC Corp.).

We fabricated three actuator-channel units with the sizes given in Table 1. While the channel length and cross-sectional area was identical for all samples, we varied the hinge chamber size $(l, w$ and $h)$ to test different actuator sizes: sample 1 thinwide, sample 2 thick-narrow and sample 3 thick-wide.

\section{A. Blocked Force Test}

To determine actuator sample forces at different applied pressures and compare them with the calculated ones using the model, we performed a blocked force test as in Fig. 5a. Each sample is mounted onto the force setup and tested in blocked condition at different folding angles. Fig. 5 displays the experimental results versus the model for $-60 \mathrm{kPa}$ and $-80 \mathrm{kPa}$ vacuum pressure. The model includes the top and bottom membrane stiffness given in (5) verified by loading the actuators with the force sensor in the direction of rotation. It is clear that the actual behavior is similar to the expected for all samples, with the model slightly greater than the measured 
data, which we suspect is due to the omitted stiffness of the side membranes. Moreover, there is a considerable discrepancy at lower angles for the largest sample 3 in Fig. 5d, which is due to the side wall force $F_{s}$ at small folding angles that collapses the bottom membrane, resulting in large stiffness ratio between the top and bottom membranes $(\mathrm{k} \gg 1)$. In addition, some air leakage could still be persevering that could cause pressure drops inside the hinge chamber.

TABLE I

THE ACTUATOR SAMPLES WITH DIFFERENT GEOMETRIC PARAMETERS TESTED ON THE FORCE MEASUREMENT SETUP.

\begin{tabular}{|c||c||c||c|}
\hline Samples & $1(\mathrm{~mm})$ & $\mathrm{h}(\mathrm{mm})$ & w $(\mathrm{mm})$ \\
\hline Sample 1 & 2 & 2.2 & 7 \\
\hline Sample 2 & 4 & 4.5 & 7 \\
\hline Sample 3 & 4 & 4.5 & 15 \\
\hline
\end{tabular}

\section{B. Shape Reconfiguration by Self-Folding}

1) Miura-Ori Tessellation: To verify the applicability of our method to more complex origami patterns, we fabricated and tested the Miura-ori tessellation depicted in Fig. 1. The pattern comprises a matrix of $2 \times 2$ base units with four hinges connected in closed chain: three for mountain and one for valley fold, or vice versa. The angular orientations of the hinges make up the wrinkles that fold out of plane.

We applied a negative pressure of $-80 \mathrm{kPa}$ to a single source port to achieve shape reconfiguration. When activated, the pressure distributes inside the actuator-channel network. Owing to reduced actuator volume, the sheet self-folding occurs in less than a second. It can also restore its flat shape at similar rates when the pressure is removed.

2) Box Sequential Self-Folding: To demonstrate the selffolding capability with variable folding sequence, we fabricated and tested a self-folding box prototype given in Fig. 1. The pattern consists of four square tiles branching out of a central tile that houses a pressure source port. We designed two hinge chambers per actuator to fold the walls to $90^{\circ}$. Two opposite walls connect to the pressure source via a 10 $\mathrm{mm}$ long macro-channel with cross-sectional dimensions of a $=0.5 \mathrm{~mm}$ and $\mathrm{b}=1 \mathrm{~mm}$, whereas the other pair embeds a micro-channel with the same length and cross-sectional size $\mathrm{a}=0.125 \mathrm{~mm}$ and $\mathrm{b}=0.5 \mathrm{~mm}$. To highlight the folding time difference between the channels visually, we introduced pin and slot connectors on the walls with macro- and microchannels, respectively. The box locks only if the pin connector folds first and then the slot to ensure their engagement.

We measured the folding time for both walls with macroand micro-channels using a high-speed camera (RX100 IV, Sony Corp.) at 1000 frames per second frame rate and plotted in Fig. 6. It shows that the walls reach $90^{\circ}$ at different rates with the pins folding first and the slots delayed for around 100 $\mathrm{ms}$. The plot is truncated below $500 \mathrm{~ms}$ as there was no time delay between the walls in the beginning.

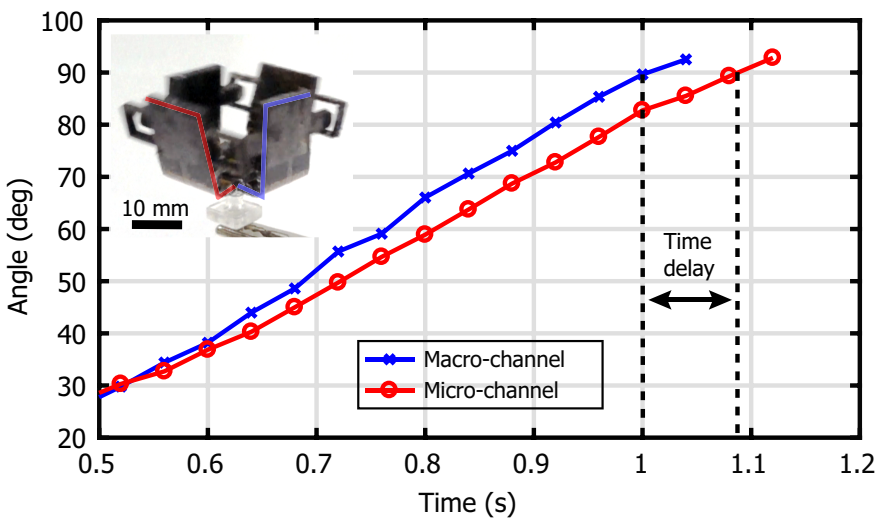

Fig. 6. The angular rotation versus time plots for folding walls with macroand micro-channels. The difference in folding time is reflected on the slopes of the curves: the wall the with macro-channel has a steeper slope and folds first, whereas the wall with the micro-channel is slower by around $100 \mathrm{~ms}$. As there was no apparent time difference for both channels before $500 \mathrm{~ms}$, the plot was truncated to highlight the time delay.

\section{CONCLUSION AND Discussion}

In this paper, we present a method for designing underactuated, programmable, fluidic actuator and channel-embedded networks for origami sequential self-folding. Our low-profile, lightweight and compact vacuum-powered folding actuators enable distribution in complex multi-hinged origami patterns with high-speed and repeatable shape transformations. We demonstrate the programmability of the proposed fluidic networks to achieve variable folding sequences of the actuators and present analytic models. Owing to layer-by-layer rapid manufacturing process, our approach allows for selective embedment of actuator-channel layers for generating mountain and valley folds with versatile motion. We showcase the efficacy of our method by testing several self-folding prototypes, including individual folding actuator units with different geometry, as well as their integration in Miura-ori and sequentially folding box patterns, and compare them to the theoretical model. We aim to further study a systematic distribution of the networks to maximize the actuation efficiency.

\section{ACKNOWLEDGMENT}

This work is supported by the SNSF "START" Project, the Swiss National Center of Competence in Research (NCCR) Robotics and the Sustainable Design of 4D Printed Active Systems (SD4D) funds.

\section{REFERENCES}

[1] Fernandes, R. and Gracias, D.H., 2012. Self-folding polymeric containers for encapsulation and delivery of drugs. Advanced drug delivery reviews, 64(14), pp.1579-1589.

[2] Yao, S., Georgakopoulos, S.V., Cook, B. and Tentzeris, M., 2014, June. A novel reconfigurable origami accordion antenna. In Microwave Symposium (IMS), 2014 IEEE MTT-S International (pp. 1-4). IEEE.

[3] Miura, K., 1985. Method of packaging and deployment of large membranes in space. Title The Institute of Space and Astronautical Science Report, 618, p.1.

[4] Zhakypov, Z. and Paik, J., 2018. Design methodology for constructing multimaterial origami robots and machines. IEEE Transactions on Robotics, 34(1), pp.151-165. 
[5] Hawkes, E., An, B., Benbernou, N.M., Tanaka, H., Kim, S., Demaine, E.D., Rus, D. and Wood, R.J., 2010. Programmable matter by folding. Proceedings of the National Academy of Sciences, 107(28), pp.1244112445.

[6] Paik, J. K., Byoungkwon, A., Rus, D., and Wood, R. J. (2012). Robotic Origamis: Self-morphing Modular Robot. In ICMC (No. EPFL-CONF206919).

[7] Bertoldi, K., Vitelli, V., Christensen, J. and van Hecke, M., 2017. Flexible mechanical metamaterials. Nature Reviews Materials, 2(11), p.17066.

[8] Faber, J.A., Arrieta, A.F. and Studart, A.R., 2018. Bioinspired spring origami. Science, 359(6382), pp.1386-1391.

[9] Belke, C.H. and Paik, J., 2017. Mori: a modular origami robot. IEEE/ASME Transactions on Mechatronics, 22(5), pp.2153-2164.

[10] Zhakypov, Z., Huang, J.L. and Paik, J., 2016. A novel torsional shape memory alloy actuator: Modeling, characterization, and control. IEEE Robotics \& Automation Magazine, 23(3), pp.65-74.

[11] Deng, D., Yang, Y., Chen, Y., Lan, X. and Tice, J., 2017. Accurately controlled sequential self-folding structures by polystyrene film. Smart Materials and Structures, 26(8), p.085040.

[12] An, N., Li, M. and Zhou, J., 2016. Predicting origami-inspired programmable self-folding of hydrogel trilayers. Smart Materials and Structures, 25(11), p.11LT02.

[13] Paik, J.K. and Wood, R.J., 2012. A bidirectional shape memory alloy folding actuator. Smart Materials and Structures, 21(6), p.065013.

[14] Mao, Y., Yu, K., Isakov, M.S., Wu, J., Dunn, M.L. and Qi, H.J., 2015. Sequential self-folding structures by 3D printed digital shape memory polymers. Scientific reports, 5, p.13616.

[15] Liu, Y., Shaw, B., Dickey, M.D. and Genzer, J., 2017. Sequential selffolding of polymer sheets. Science Advances, 3(3), p.e1602417.

[16] Lee, Y., Lee, H., Hwang, T., Lee, J.G. and Cho, M., 2015. Sequential folding using light-activated polystyrene sheet. Scientific reports, 5, p. 16544 .

[17] Laflin, K.E., Morris, C.J., Muqeem, T. and Gracias, D.H., 2012. Laser triggered sequential folding of microstructures. Applied Physics Letters, 101(13), p.131901.
[18] Niiyama, R., Sun, X., Sung, C., An, B., Rus, D. and Kim, S., 2015. Pouch motors: Printable soft actuators integrated with computational design. Soft Robotics, 2(2), pp.59-70.

[19] Russo, S., Ranzani, T., Gafford, J., Walsh, C.J. and Wood, R.J., 2016, May. Soft pop-up mechanisms for micro surgical tools: Design and characterization of compliant millimeter-scale articulated structures. In Robotics and Automation (ICRA), 2016 IEEE International Conference on (pp. 750-757). IEEE.

[20] Overvelde, J.T., De Jong, T.A., Shevchenko, Y., Becerra, S.A., Whitesides, G.M., Weaver, J.C., Hoberman, C. and Bertoldi, K., 2016. A threedimensional actuated origami-inspired transformable metamaterial with multiple degrees of freedom. Nature communications, 7, p.10929.

[21] Li, S., Vogt, D.M., Rus, D. and Wood, R.J., 2017. Fluid-driven origamiinspired artificial muscles. Proceedings of the National Academy of Sciences, p.201713450.

[22] Hibbeler, R.C. and Kiang, T., 2015. Structural analysis. Pearson Prentice Hall. 